\title{
Laser Guiding at Relativistic Intensities and Wakefield Particle Acceleration in Plasma Channels
}

\author{
C.G.R. Geddes ${ }^{1,2}$, Cs. Toth ${ }^{1}$, J. Van Tilborg ${ }^{1,3}$, E. Esarey ${ }^{1}$, \\ C.B. Schroeder ${ }^{1}$, D. Bruhwiler ${ }^{4}$, J. Cary ${ }^{4,5}$, W.P. Leemans ${ }^{1}$ \\ ${ }^{1}$ Lawrence Berkeley National Laboratory, 1 Cyclotron Rd, Berkeley CA 94720 \\ ${ }^{2}$ University of California, Berkeley, Berkeley CA 94720 \\ ${ }^{3}$ Technische Universiteit Eindhoven, Postbus 513,5600 MB Eindhoven, the Netherlands. \\ ${ }^{4}$ Tech-X Corporation, 5621 Arapahoe Ave. Suite A, Boulder CO, 80303 \\ ${ }^{5}$ University of Colorado, Boulder CO, 80309
}

\begin{abstract}
Electron beams with hundreds of picoCoulombs of charge in percent energy spread at above $80 \mathrm{MeV}$, and with few milliradian divergence, have been produced for the first time in a high gradient laser wakefield accelerator by guiding the drive laser pulse. Channels formed by hydrodynamic shock were used to guide acceleration relevant laser intensities of at least $1 \mathrm{E} 18 \mathrm{~W} / \mathrm{cm}^{2}$ at the guide output over more than 10 Rayleigh lengths at LBNL's l'OASIS facility $\left(10 \mathrm{TW}, 2 \mathrm{E} 19 \mathrm{~W} / \mathrm{cm}^{2}\right)$. The pondermotive force of the laser pulse drove an intense plasma wave, producing acceleration gradients on the order of $100 \mathrm{GV} / \mathrm{m}$. Electrons were trapped from the background plasma and accelerated. By extending the acceleration length using the guiding channel, the energy of the electron beam was greatly increased, and bunches of small energy spread and low emittance were formed. Experiments varying gas jet length as well as simulations indicate that the high quality beams were formed when beam loading turned off injection after an initial load, producing an isolated bunch, and when that bunch was subsequently accelerated to the dephasing length at which point it rotated in phase space to produce low energy spread.
\end{abstract}

\section{INTRODUCTION}

Laser wakefield acceleration experiments to date have demonstrated that electrons can be accelerated by plasma waves with an electric field up to hundreds of $\mathrm{GV} / \mathrm{m}[1$ $3]$, indicating the potential for more compact accelerators. Acceleration distance and hence electron beam energy and quality has been limited however by the difficulty of retaining high laser intensity over a long distance of propagation in the plasma, resulting in a poor quality electron bunch with $100 \%$ energy spread and an exponentially small fraction of electrons at high energy[1-3]. Laser power in these experiments was above the critical power for self-focusing and the laser pulse length exceeded the plasma wavelength. In this self modulated regime, some self-guiding of the laser pulse occurs due to relativistic modification of the plasma refractive index, but the laser pulse is highly unstable $[4,5]$, limiting propagation length to little more than a diffraction range $Z_{R}[6]$. The best results have hence been obtained by increasing 
spot size to increase $Z_{R}$, requiring ever greater laser power, but this approach has still been limited to distances of a few hundred micron[6]. To circumvent this limit and to realize the potential of laser accelerators, the laser pulse must be guided at relativistic intensities, and its propagation controlled over distances of several mm or greater [7].

\section{EXPERIMENT}

We report the first guiding of relativistic laser intensities over many diffraction ranges by a plasma channel, and the resulting production of electron bunches of few percent energy spread, hundreds of picoCoulombs of charge, and milliradian divergence at energies above $80 \mathrm{MeV}$. The high acceleration gradients of previous laser accelerator experiments are retained over longer distances, and the beam quality is comparable to state of the art RF accelerators. Experiments and supporting simulations indicate that the high quality bunches are formed from self trapped electrons when beam loading turns off self trapping after the loading of an initial bunch, and when the bunch is accelerated to the dephasing length over which trapped electrons outrun the wake.

Guiding at high intensities to produce a channel guided accelerator required controlling for both diffraction and plasma effects. Previous experiments demonstrated guiding for input pulse intensities at up to $2 \mathrm{E} 17 \mathrm{~W} / \mathrm{cm}^{2}[8-11]$, where a parabolic transverse plasma density profile can be matched to guide the low intensity pulse[12]. At high intensities, relativistic self guiding occurs when the quiver motion of the electrons causes their mass to increase, changing the refractive index. This occurs in regimes of interest to wakefield acceleration and provides some self guiding, but is unstable $[4,5]$. The channel must hence balance both diffraction and instabilities to achieve long propagation distance at high intensity.

The multi arm l'OASIS Ti:Sapphire laser[13, 14], operating at $800 \mathrm{~nm}$ with chirped pulse amplification, was used to form the guiding channel using a variation of the ignitor heater method[9] and to drive the plasma wake (Figure $1 \mathrm{~A}$ ). A plasma was formed by an ignitor pulse $(15 \mathrm{~mJ}, 60 \mathrm{fs})$ in a $2.5 \mathrm{~mm}$ long supersonic $\mathrm{H}_{2}$ gas jet with an atomic density of $3-4 \mathrm{E} 19 \mathrm{~cm}^{-3}$, then heated by a heater pulse $(150 \mathrm{~mJ}, 250 \mathrm{ps})$. Hydrodynamic expansion of the plasma formed a channel[8] which guided the relativistically intense drive pulse focused at its edge. The drive pulse $(500 \mathrm{~mJ}, 55 \mathrm{fs})$
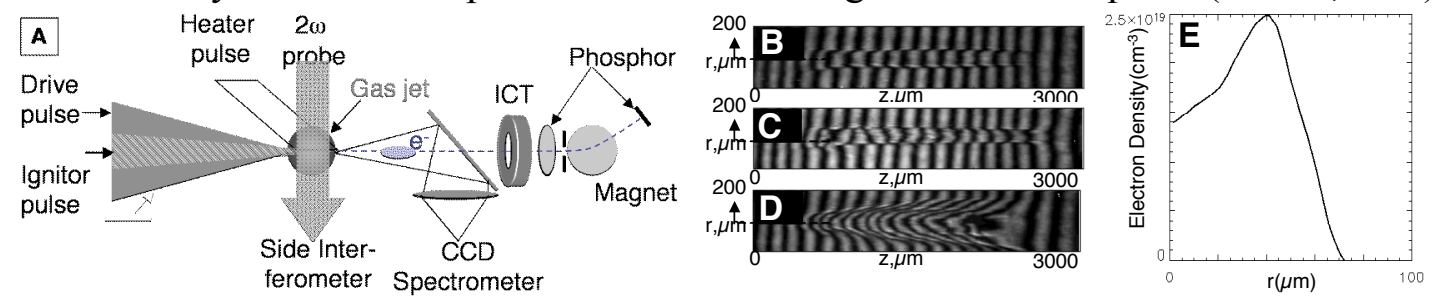

FIGURE 1. (a) Experimental setup showing the gas jet with laser beams and diagnostics. (b-d) Side interferometer images show the channel structure and laser propagation. 500ps after formation, at the time of drive pulse arrival, a channel was formed (a) and the density profile was obtained by Abel inversion (e). Image of the drive beam at $4 \mathrm{TW}$ propagating in this channel (c) is similar to (b) indicating good confinement, while the unguided beam (d) diffracts rapidly. 
was focused to a spot of 7-8.5 $\mu \mathrm{m}$ FWHM to reach intensities up to $1.1 \mathrm{E} 19 \mathrm{~W} / \mathrm{cm}^{2}$, resulting in $Z_{R} \sim 200 \mu \mathrm{m}$ so that the channel was $>10 Z_{R}$ long. Propagation of the laser was monitored with a side interferometer, mode imager $\mathrm{CCD}$, and transmitted light spectrometer. Electrons accelerated by the plasma wake of the drive beam were analyzed using an integrating current transformer (ICT), a phosphor screen, and a magnetic spectrometer.

\section{RESULTS AND SIMULATIONS}

By adjusting the timing of the beams, the channel profile has been adjusted to guide the drive pulse at various powers and to compensate for the presence of self guiding[15]. With the channel tuned to match the low power guiding condition, aberration free guiding of low power pulses $(0.5 \mathrm{TW}<$ Pcrit $=$ critical power for self guiding) was obtained with transmission efficiency of $\sim 50 \%$, but pulses above $\mathrm{P}_{\text {crit }}$ were aberrated, displaying enlarged output spot size and lowered intensity. Re-tuning the channel to slightly flatten the density profile allowed compensation for the presence of self guiding, and powers up to $4 \mathrm{TW}\left(7 \mathrm{E} 18 \mathrm{~W} / \mathrm{cm}^{2}\right)$ were guided without aberration. The channel interferogram for this tune at the time of main pulse arrival is shown in Figure 1b. Propagation of the main beam in the channel does not change the image (Figure 1c), indicating that the laser is well confined to the channel, as leakage outside the channel would ionize additional gas. The unguided beam diffracts rapidly, demonstrating that self guiding alone is insufficient to control mode propagation over this distance (Figure 1d). The plasma density profile of the channel (Figure 1e) shows a nearly parabolic transverse dependence, with a rise in density over the spot diameter $30 \%$ less than the low power matching condition reflecting the adjustment made to compensate for self guiding.

Figure 2 shows the mode images of the laser spot propagation at $4 \mathrm{TW}$ ( $7 \mu \mathrm{m}$ input spot, $7 \mathrm{E} 18 \mathrm{~W} / \mathrm{cm}^{2}$ ). With the channel on, the output spot (b) matches the input (a). The mode imager resolution is restricted by $\mathrm{f} / \#$ constraints in the target chamber so that it yields a $12 \mu \mathrm{m}$ FWHM spot size for both input and output. Hence guided intensity is between $1 \mathrm{E} 18$ and $2.5 \mathrm{E} 18 \mathrm{~W} / \mathrm{cm}^{2}$ with the lower limit set by the $12 \mu \mathrm{m}$ mode imager observation and the upper limit set by the input spot size. The vacuum output displays diffraction (c), indicating the effectiveness of the guide, and with the

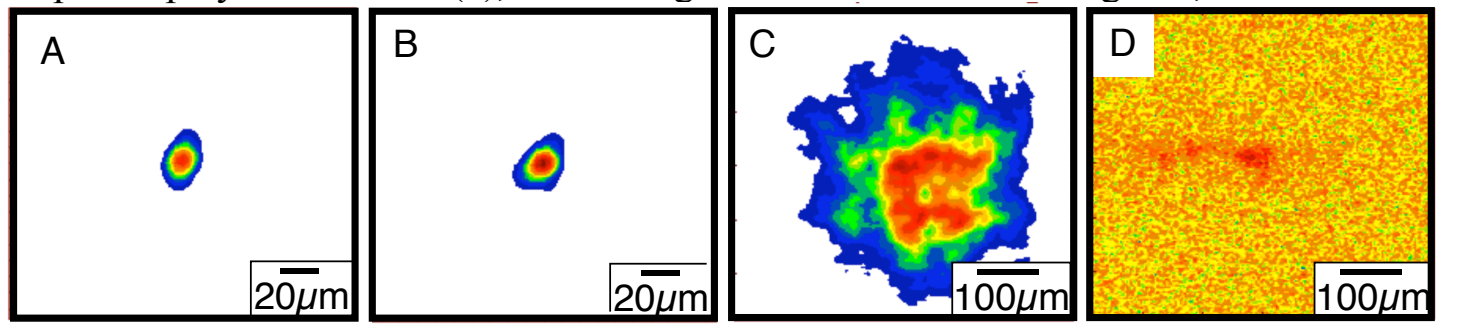

FIGURE 2. Mode images of laser propagation at $4 \mathrm{TW}$. The output image with the guide on (b) is indistinguishable from the input (a) indicating unaberrated propagation at 4 times $\mathrm{P}_{\text {crit }}$. Guide output intensity corresponds to $\mathrm{a}_{0} \sim 1$ in the relevant range for wakefield acceleration. The unguided images show diffraction in vacuum (c) and ionization enhanced diffraction with the gas jet on (d). 
gas jet on but the channel off (d) diffraction is increased by ionization effects[16, 17], showing that self guiding alone is insufficient to efficiently guide the beam.

Transmission at $4 \mathrm{TW}$ was $35 \%$, a reduction of one third from the low power case, indicating that substantial power was deposited in plasma waves. The depletion observed is consistent with particle in cell simulations run with the experimental parameters (below), which indicate that a plasma wave averaging $200 \mathrm{GV} / \mathrm{m}$ is excited in the last $0.5 \mathrm{~mm}$ of guide length. No electrons are self trapped at $4 \mathrm{TW}$, making this an attractive structure for controlled injection experiments[18, 19]. Quality and stability of laser accelerated bunches may be greatly increased by controlling injection rather than relying on instabilities such as self modulation, but such experiments have until now been hampered by lack of a long scale length high gradient structure such as the channel described here can provide. Experiments on colliding pulse injection[19] are under way in this geometry.

The transmitted spectrum of the laser pulse was also analyzed as a diagnostic of channel confinement and plasma wave excitation. Without the guiding channel, the spectrum showed severe blue shifting, which occurs when the laser ionizes the gas it is passing through. This feature was nearly eliminated for the channeled beam, indicating that the laser was well confined to the guiding channel. The spectrum was close to undistorted with transmission of the $800 \mathrm{~nm}$ feature near $30 \%$ at $4 \mathrm{TW}$, close to the transmission observed on the mode imager. A red shifted shoulder also appeared in the channeled case, increasing in relative amplitude with increasing laser power, consistent with depletion of the laser into plasma waves.

The presence of a high amplitude plasma wave was experimentally verified by increasing laser power and observing that electrons were trapped and accelerated. At $9 \mathrm{TW}$, optimal performance was found in a channel with an axial density of $1.9 \mathrm{E} 19 \mathrm{~cm}^{-3}$ and with a parabolic profile with $40 \%$ less rise in density over a spot diameter than the low power matched case. The laser was well confined to the channel at this power but some aberration was always present, with output mode sizes near $24 \mu \mathrm{m}$ FWHM, likely due to the strong self guiding present here. The drive laser pulse was a factor of two longer than the linear plasma period, i.e. in the selfmodulated regime. This regime was chosen to allow comparison to unchannelled experiments, and also because the slower phase velocity of the wake at high plasma density allowed trapping of background plasma electrons yielding high charge electron beams without a separate injector.

The channel guided accelerator produced high charge electron beams with low energy spread at high energy, and with low divergence[20]. Figure 3a shows a bunch of 3E9 electrons within an energy spread of $\pm 4 \%$ centered at $78 \mathrm{MeV}$. Due to pointing fluctuations which change the incoupling of the drive beam to the guide, this feature varied shot to shot, and bunches energy spread as low as $2 \%$ with $2 \mathrm{E} 9$ electrons at 86 $\mathrm{MeV}$ were observed, as well as bunches of 1E9 electrons at energies up to $150 \mathrm{MeV}$. The charge in the bunches was calibrated using the ICT and radionuclide activation measurements, both of which were consistent. Total charge in the electron beam was near 2E10 electrons; the low energy portion can be separated using a bend magnet, leaving a high energy high quality bunch. The divergence of the bunches near $80 \mathrm{MeV}$ was $3 \mathrm{mrad}$. This is half the divergence of the full beam observed before the magnet (Figure 3b), consistent with other experiments which have shown that the high energy 

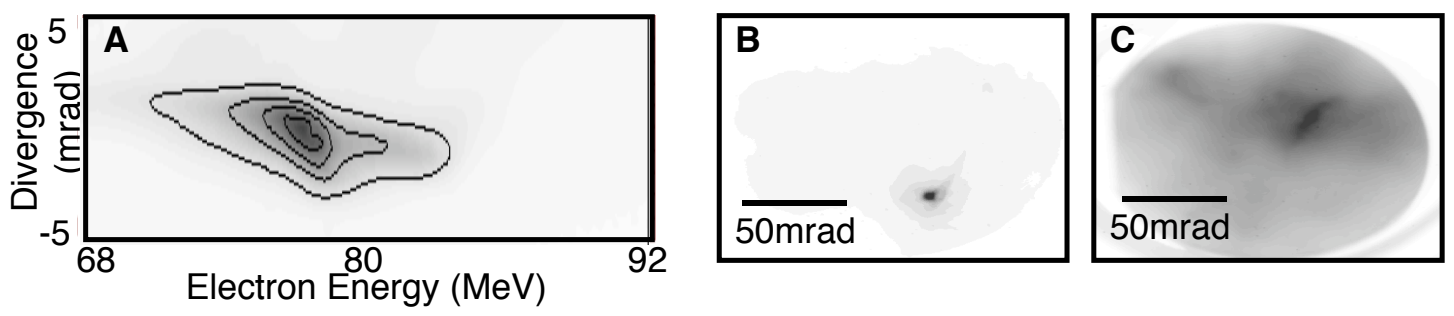

FIGURE 3. Electron bunches. The electron energy spectrum of the channeled accelerator (a) shows the appearance of monoenergetic features, here with 3E9 electrons in a bunch with energy spread of 4\% FWHM at $78 \mathrm{MeV}$. Divergence was near $3 \mathrm{mrad}$ FWHM for this bunch (a), and 6 mrad for the whole beam (b). The unguided accelerator in the same gas jet by contrast shows a nearly smooth exponenetial spectrum with a few MeV temperature (not shown), and much wider divergence (c).

portion of the beam is better collimated[2]. The normalized geometric emittance obtained from assuming the bunch comes from a source $\sim$ the laser spot size is $1-2 \pi$ mm-mrad, competitive with state of the art radiofrequency facilities.

The accelerator was operated in the same gas jet without the guiding channel. Density was separately optimized for the unchanneled accelerator and best performance was at $\mathrm{n}_{\mathrm{e}}=4 \mathrm{E} 19 \mathrm{~cm}^{-3}$. This produced an exponential energy distribution with a $2.6 \mathrm{MeV}$ temperature below $10 \mathrm{MeV}$ and an $8 \mathrm{MeV}$ temperature above $10 \mathrm{MeV}$. No electrons were observed (detection threshold $\sim 10^{7}$ electrons on phosphor) above $40 \mathrm{MeV}$. The bulk of the distribution was smooth, with occasional structure in the tail of the distribution containing $<2 \%$ of the charge. The beam divergence (Figure $3 \mathrm{c}$ ) is much larger than the channeled case. No difference was observed between operation in a neutral gas jet and a pre-ionized (but not channeled) plasma, indicating that channeling and not ionization is the important effect.

Two dimensional particle in cell simulations performed with parameters close to the experiment using the code VORPAL[21] show formation of bunches similar to those observed in the channeled accelerator. If laser pulse strength was just above that required to self trap electrons, loading of the wake[22] by the initial electron bunch trapped suppressed further injection. This lead to a bunch of electrons isolated in phase space (Figure 4 a). If this bunch was accelerated until it dephased from the
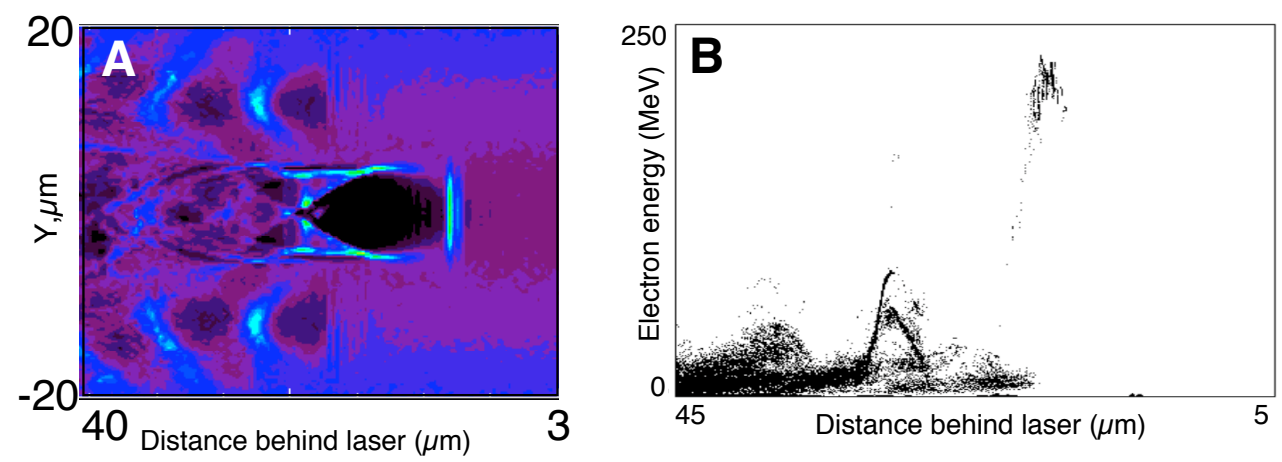

FIGURE 4. Particle in cell simulations show that a high quality bunch is formed when beam loading turns off injection, resulting in a bunch isolated in phase space. This is visible in the density contour (a), where the wake amplitude is lowered following the bunches visible in the first two buckets. These particles are then concentrated in energy at the dephasing length (b) forming a low energy spread bunch. 
wake, the leading edge of the bunch was decelerated while the tail was accelerated, concentrating the particles in energy and forming a low energy spread bunch at the dephasing length (Figure 4b). Matching accelerator length to the dephasing length for the jet length and $Z_{R}$ used required a guiding channel.

The dephasing condition can alternately be met, though less efficiently, without a channel. A short gas jet can be used at the cost of reduced final energy, or self guiding can be used but this is much less stable than a channel A large laser spot can also be used to obtain a long $Z_{R}$ at the cost of efficiency (higher laser power/high energy electron). To evaluate the impact of dephasing on the accelerator experimentally, gas jets of variable length and density were used without channeling[23]. PIC simulations were used to evaluate the dephasing length, since analytic estimates are not valid in this regime. With the gas jet lengths and laser $Z_{R}$ available, it was possible to test at and after dephasing at a density of 4E19, as well as before and after dephasing at a density of 2E19. The electron beam spectra (Figure 5) show that extraction of the beam before dephasing $\left(600 \mu \mathrm{m}\right.$ plasma at $\left.2 \mathrm{E} 19 \mathrm{~cm}^{-3}\right)$ yielded low energies. Analysis of individual phosphor images (inset) showed that the distribution was smooth. Extraction after dephasing $\left(4 \mathrm{~mm}\right.$ at $2 \mathrm{E} 19 \mathrm{~cm}^{-3}$, or $2 \mathrm{~mm}$ at $\left.4 \mathrm{E} 19 \mathrm{~cm}^{-3}\right)$ produced high energies with some structure in the distribution. However, the highest energies for a given density, as well as the most monoenergetic features present in phosphor images were obtained when the beam was extracted at the dephasing length $(600 \mu \mathrm{m}$ at $\left.4 \mathrm{E} 19 \mathrm{~cm}^{-3}\right)$. This demonstrates the importance of tuning the accelerator to the dephasing length. Even with optmization for the dephasing length however, the unchanneled accelerator produced less stable lower quality beams than the channeled accelerator, indicating the advantage of controlling and extending laser propagation length using the channel.

\section{CONCLUSIONS}

Experiments have demonstrated guiding of relativistically intense laser pulses over many $Z_{R}$ in plasmas, and tailoring of the plasma profile to provide guiding without
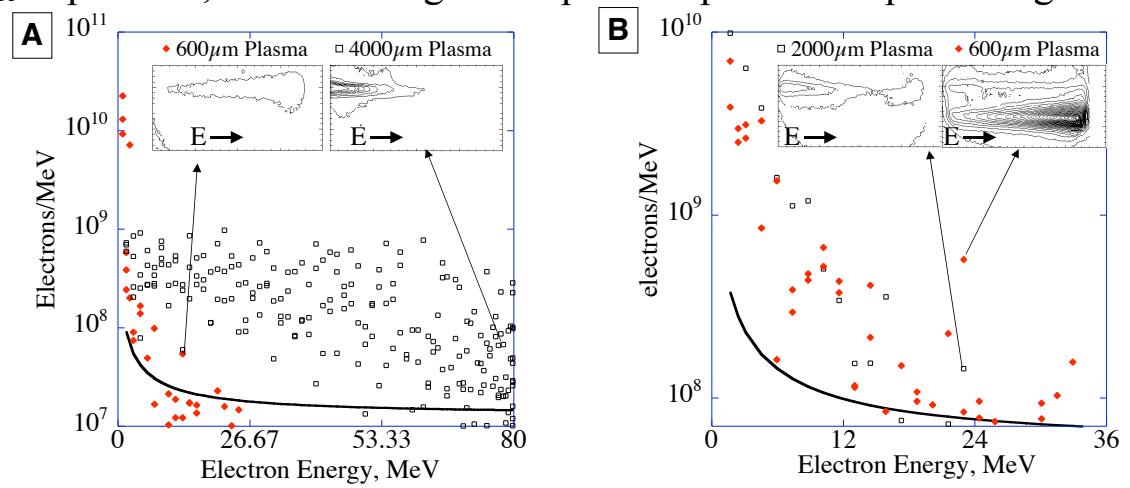

FIGURE 5. Variation of gas jet length confirms the importance of dephasing. At $n_{e}=2 e 19$, extraction of the beam before the dephasing length of $1.5 \mathrm{~mm}$ resulted in a low energy spectrum with little structure, while extraction at or after dephasing produced higher energies with more monoenergetic features (a). At $\mathrm{n}_{\mathrm{e}}=4 \mathrm{e} 19$, no additional acceleration was observed beyond the dephasing length of $600 \mu \mathrm{m}$ and structure was reduced (b). 
detectable aberration up to several times the relativistic self guiding threshold[15]. Input intensities near $10^{19} \mathrm{~W} / \mathrm{cm}^{2}$ have been guided without self injection of electrons. Increasing density and intensity produced self trapped electron beams of percent energy spread with several $10^{9}$ electrons and with emittance comparable to state of the art radio frequency accelerators[20]. This offers the possibility of new classes of experiments on laser driven accelerators and indicates that development of high energy high quality beams is feasible using this method, benefiting many applications. Experiments and simulations indicate that these beams are formed by a combination of beam loading and dephasing[23]. Controlled injection using the colliding pulse method[19] will be tried in this structure, which may further stabilize and improve the bunch quality.

\section{ACKNOWLEDGMENTS}

Work supported by the U.S. Dept. of Energy contract DE-AC03-76SF00098, and by the Office of Advanced Scientific Computing Research under the Scientific Discovery through Advanced Computing (SciDAC) Program. This research used resources of the National Energy Research Scientific Computing Center, which is supported by the Office of Science of the U.S. Department of Energy under Contract No. DE-AC03-76SF00098. C.Geddes is also supported by the Hertz foundation. C. Geddes acknowledges his faculty advisor Jonathan Wurtele, at UC Berkeley. We appreciate contributions from Gerry Dugan, Jerome Faure, Gwenael Fubiani, Bob Nagler, Kei Nakamura, Ned Saleh, Brad Shadwick, Leon Archambault, Michael Dickinson, Scott Dimaggio, Don Syversrud, Joe Wallig, and Nathan Ybarrolaza.

\section{REFERENCES}

1. A. Modena et al., Nature 377, 606 (1995).

2. V. Malka et al., Science 298, 1596 (2002).

3. W. P. Leemans et al., Phys. Rev. Lett. 8917, 4802 (2002).

4. E. Esarey, J. Krall, P. Sprangle, Phys. Rev. Lett. 72, 2887 (1994).

5. E. Esarey, P. Sprangle, J. Krall, A. Ting, IEEE J. Quantum Electron. 33, 1879 (1997).

6. Z. Najmudin et al., Phys. Plasmas 10, 2071 (2003).

7. E. Esarey, P. Sprangle, J. Krall, A. Ting, IEEE Trans. Plasma. Sci. 24, 252 (1996).

8. C. G. Durfee, H. M. Milchberg, Phys. Rev. Lett. 71, 2409 (1993).

9. P. Volfbeyn, E. Esarey, W. P. Leemans, Phys. Plasmas 6, 2269 (1999).

10. K. Y. Kim, I. Alexeev, J. Fan, E. Parra, H. M. Milchberg, paper presented at the AIP Conf. Proc., 647, 646 (2002).

11. E. W. Gaul et al., Appl. Phys. Lett. 77, 4112 (2000).

12. P. Sprangle, E. Esarey, J. Krall, G. Joyce, Phys. Rev. Lett. 69, 2200 (1992).

13. C. Toth et al., SPIE Proceedings 5448 (2004).

14. W. P. Leemans et al., Phys. Plasmas 5, 1615 (1998).

15. C. G. R. Geddes et al., Submitted to Phys. Rev. Lett., 2004.

16. R. Rankin, C. E. Capjack, N. H. Burnett, P. B. Corkum, Opt. Lett. 16, 835 (1991).

17. W. P. Leemans et al., Phys. Rev. A 46, 1091 (1992).

18. D. Umstadter, J. K. Kim, E. Dodd, Phys. Rev. Lett. 76, 2073 (1996).

19. E. Esarey, R. F. Hubbard, W. P. Leemans, A. Ting, P. Sprangle, Phys. Rev. Lett. 79, 2682 (1997).

20. C. G. R. Geddes et al., Accepted for publication in Nature, 2004.

21. C. Nieter, J. R. Cary, J. Comp. Phys. 196, 448 (May 20 2004, 2004).

22. T. Katsouleas, S. Wilks, S. Chen, J. M. Dawson, J. J. Su, Particle Accelerators 22, 81 (1987, 1987).

23. C. G. R. Geddes et al., Submitted to Phys. Rev. Lett., 2004. 